\title{
Concomitant identification of subspecies and insecticide resistance-associated mutations in the mosquito Culex pipiens complex by primer extension-based genotyping
}

\author{
Osamu Komagata ${ }^{1)}$, Shinji Kasai ${ }^{1)}$, Itsumi Obara ${ }^{1), 2)}$, Naoki Motoyama ${ }^{2)}$, \\ Ikuo TANaKa ${ }^{3)}$ Mutsuo Kobayashi ${ }^{1)}$ and Takashi Tomita ${ }^{1), 4)}$ \\ 1) Department of Medical Entomology, National Institute of Infectious Diseases, \\ Toyama 1-23-1, Shinjuku-ku, Tokyo, 162-8640 Japan \\ 2) Laboratory of Pesticide Toxicology Graduate School of Horticulture, \\ Chiba University, Matsudo 648. Matsudo, Chiba, 271-8510 Japan \\ 3) Department of Environmental Biology, Japan Environmental Sanitation Center, \\ Yotsuyakamicho 10-6, Kawasaki-ku, Kawasaki, Kanagawa, 210-0828 Japan \\ 4) E-mail: tcmita@nih.go.jp
}

(Received: 10 December 2007; Accepted: 36 February 2008)

\begin{abstract}
Management of insecticide resistance in the mosquito Culex pipiens complex is important because this complex is a major vector of lymphatic filariasis and West Nile fever, which occurs worldwide, and has been exposed to frequent chemical controls with a variety of insecticides. We applied the SNaPshot assay, a primer extension-based genotyping method, to the identification of both insecticide resistance-associated point mutations at insecticidal active sites and the mosquito subspecies distributed in Japan. Variations at Leu1014 and Val1016 positions in the sodium channel and Ser119 in Drosophila Ace-paralogous acetylcholiesterase (p-Ace) were individually tested for the detection of mutations conferring decreased sensitivity to nerve insecticidal targets. Moreover, sequence differentiations in Drosophila Ace-orthologous acetylcholinesterase (o-Ace) gene were concomitantly tested for the detection of subspecies of $C x$. pipiens pallens, $C x$. p. molestus and $C x$. quinquefasciatus. The exactness of the SNaPshot assay was verified using standard laboratory strains and mutagenized synthesized DNA. The assay was then applied to laboratorycolonized mosquitoes that were captured from Kyushu and Honshu, Japan. Judgments for both $C x . p . m$. and $C x$. p. pallens by SNaPshot assay agreed with the respective autogenicities of the colonies tested, except for an erroneous individual. Mutation of Leu1014 to Phe and Ser in the sodium channel genes were specifically identified in the samples of $C x . p . m$. and $C x$. p. pallens., respectively. No mutation was detected for the other target sites.
\end{abstract}

Key words: Culex pipiens complex, molecular taxonomy, insecticide resistance, SNPs typing, sodium channel, acetylcholinesterase

\section{INTRODUCTION}

The mosquito Culex pipiens complex is a major vector of lymphatic filariasis and

\footnotetext{
Nomenclatures: Gene symbols for o-Ace, p-Ace, and $\mathrm{SC}$ proteins are shown by $0-A c e, p-A c e$, and $S C$ in italics, respectively.
}

West Nile fever (WNF) and is distributed worldwide. The classification and nomenclature of the species complex have been historically controversial (Harbach et al., 1984; Vinogradova, 2000). The mosquitoes distributed in the Northern Hemisphere are now mostly classified into four subspecies (or forms/sibling species) on the basis of the multiple traits of 
morphological variations, physiological or behavioral properties, and distributional areas (Tanaka et al., 1979): Culex pipiens pipiens (L.), Cx. p. molestus (Forskal) (Cx. p. $m$.), $C x$. p. pallens (Coquillet), and $C x$. quinquefasciatus (Say) (Cx.q.). Overall, the major aboveground habitants of $C x$. $p$. complex in temperate zones of the Northern Hemisphere switch between $C x$. $p$. pallens in East Asia and $C x$. p. pipiens in Europe and North America (Tanaka et al., 1979). Culex. p. pipiens has not been identified in Japan (Sasa, 1971; Tanaka et al., 1979; Tanaka, 2005). Culex. p. pallens and a principally underground habitant Cx. p. m. (Ishii, 1978) are distributed in the mainislands of Japan, while an aboveground habitant $C x . q$. is distributed in the Nansei Islands, parts of Kyushu and Shikoku districts of Japan (Tanaka et al., 1979). Actually, the distributions, habitats and occurrence of the three subspecies partially overlap in Japan. Studies on the host-feeding habits of each subspecies are prerequisite to locally evaluate the vector competence of the $C x$. $p$. species complex in terms of the invasion of West Nile virus because of a ceaseless epidemic of WNF in North America since 1999.

Molecular taxonomic techniques were explored, targetting interspecific variations in Drosophila Ace-orthologous acetylcholinesterase (o-Ace) gene sequences of $C x$. p. pipiens and $C x . q$. (Bourguet et al., 1998; Aspen and Savage, 2003). Smith and Fonseca presented an allele specific PCR (AS-PCR) assay (Smith and Fonseca, 2004). The assay sorts the $C x$. $p$. complex into three classes: $C x$. p. pallens, $C x$. $q$., and an inseparable class including $C x . p$. pipiens and $C x . p$. $m$. Kasai pointed out substantial defects in the primer design presented by Smith and Fonseca and alternatively demonstrated the availability of modified primers for the same objective (Kasai et al., 2008).

Insecticide groups such as organophosphates, pyrethroids, insect growth regulators and Bacillus biotic pesticides have been globally utilized to control $C x$. p. mosquitoes. As a result, single or complex insecticide-resistance has developed in local populations of the $C x$. $p$. complex (Ferre and Van Rie, 2002; Sharma et al., 2004; Hamadan et al., 2005; Nazni et al., 2005; Vatandoost et al., 2005; Casimiro et al., 2006; Cui et al., 2006; Etang et al., 2006; Tia et al., 2006). A recent investigation revealed that the development of larval resistance to the pyrethroid etofenprox was prominent especially in the Cx. p. $m$. populations of Japan (Kasai et al., 2007) . Larval resistance to an organophosphorus fenitrothion in a field colony of $C x . p . m$. in Tokyo was also reported at an earlier date (Kawakami, 1989).

p-Ace (Drosophila Ace-paralogous acetylcholinesetrase) and sodium channel (SC) are the sites of action for organophosphorous and pyrethroid compounds, respectively. The mutations that are conferring or associated with insecticide resistance in these genes have been studied in Cx. p. mosquitoes (Weill et al., 2003; Davies et al., 2007). Gly119Ser in p-Ace confers carbamate and organophosphorus insecticide resistance due to a insensitive nerve target. This mutation was found in resistant strains of $C x$. p. pipiens and $C x . q$. of European and African origins (Weill et al., 2003). An amino acid substitution mutation, Leu1014Phe, in SC was originally found in houseflies with knockdown resistance $(k d r)$ and this mutation conferred a decreased nerve sensitivity to pyrethroids (Miyazaki et al., 1996; Williamson et al., 1996). Equivalent amino acid substitutions have also been identified from DDT- and pyrethroid-resistant insects from a variety of medical and agricultural pest species (Tomita et al., 2005), including Cx. q. (Martinez-Torres et al., 1999; Xu et al., 2005). In addition to the typical Phe $1014 k d r$ mutation, another substitution to Ser occurring at the same position was reported as a resistance-associated atypical $k d r$ mutation in $C x . p$. mosquitoes (Martinez-Torres et al., 1999) (where the subspecies is undiscerned). Val1016 Gly was first found in a strain of Aedes aegypti 
(Brengues et al., 2003), but the association of the mutation with insecticide resistance was not tested. An equivalent mutation is also involved in a pyrethroid-resistant JPP-B strain of Saudi Arabian origin Cx. $q$. (Kasai et al., 1998). The frequency distribution of insecticide-resistant genes can serve as basic information for local resistance-management. However, the resistance-associated mutations described above have not been systematically censused in the natural populations of the $C x$. p. complex in Japan.

Molecular genotyping for monitoring insecticide resistance has several advantages over authentic bioassay because genotyping makes it possible to determine physiological or biochemical mechanisms and thus easier to infer potential crossresistance. Genotyping can also directly test individual field-collected insects, irrespective of their developmental stages and whether the insects are alive or dead. Molecular genotyping consequently provides the maximum effective sampling size, compared to bioassays that ordinarily require laboratory colonization, during which genetic variation is lost especially for species difficult to breed. Finally, it is possible to identify heterozygous recessive resistant mutations even if the frequency is too low to impair insecticidal efficacy in a local population.

In this study, we adapted a SNaPshot assay to the concomitant molecular detection of subspecies and resistance-associated point mutations in $C x$. $p$. mosquitoes, targetting o-Ace. p-Ace and SC genes, and subsequently applied the technique to recent laboratory colonies of Japanese origin.

The SNaPshot assay using a commercial kit was used to detect single nucleotide polymorphisms (SNPs). The protocol relies on the extension of multiple specific minisequencing primers toward the 3 ' direction by single dideoxy-NTPs that are fluorescence labeled. The sites of the added dideoxy-NTPs correspond to the respective target SNP sites in the DNA tem- plates tested. The SNPs are detectable by the electrophoretic separations of the extended minisequencing primers on a DNA sequencer.

\section{Materials and Methods}

\section{Standard mosquito strains}

Four strains of $C x$. pipiens complex were used as standards for the SNaPshot analysis. OGS is an insecticide susceptible strain of $C x$. $q$., originating from Chichijima, Ogasawara Islands, Japan (Kono and Tomita, 1992). JPP-B is a derivative of the JPal-per strain of $C x . q$. that originated from Saudi Arabia and is highly resistant to permethrin due to decreased target sensitivity and cytochrome P450 monooxygenase-mediated detoxification (Kasai et al., 1998). SBY strain was derived after larval selection with etofenprox from a laboratory colony of $C x . p . m$. Shibuya that originated from Shibuya-ku, Tokyo (Kasai et al., 2008). RNS strain was derived after larval selection with etofenprox from a laboratory colony of Cx. p. pallens Rinshi that originated from Shinagawa-ku, Tokyo (Kasai et al., 2008). JPP-B, SBY and RNS strains showed a similar level of larval resistance to etofenprox at a $10^{3}$ order resistance ratio (Kasai, unpublished). Resistance-conferring (or -associated) amino acid substitution mutations, Val1016 Gly, Leu1014Phe and Leu 1014 Ser in the SC gene were fixed within the JPP-B, SBY and RNS strains, respectively (Table 1), Numbering of the amino acid positions followed that of the housefly (Williamson et al., 1996). Males from these strains were used as standards for the respective subspecies in the molecular analysis.

\section{Synthesized standard DNA}

Because of unavailability of a strain with insecticide resistance-conferring the Gly 119Ser mutation the in p-Ace gene (the numbering follows the standard protein sequence of acetylcholinesterase), a DNA fragment of 189 bp carrying this mutation 
Table 1. Laboratory strains and their SNaPshot targets.

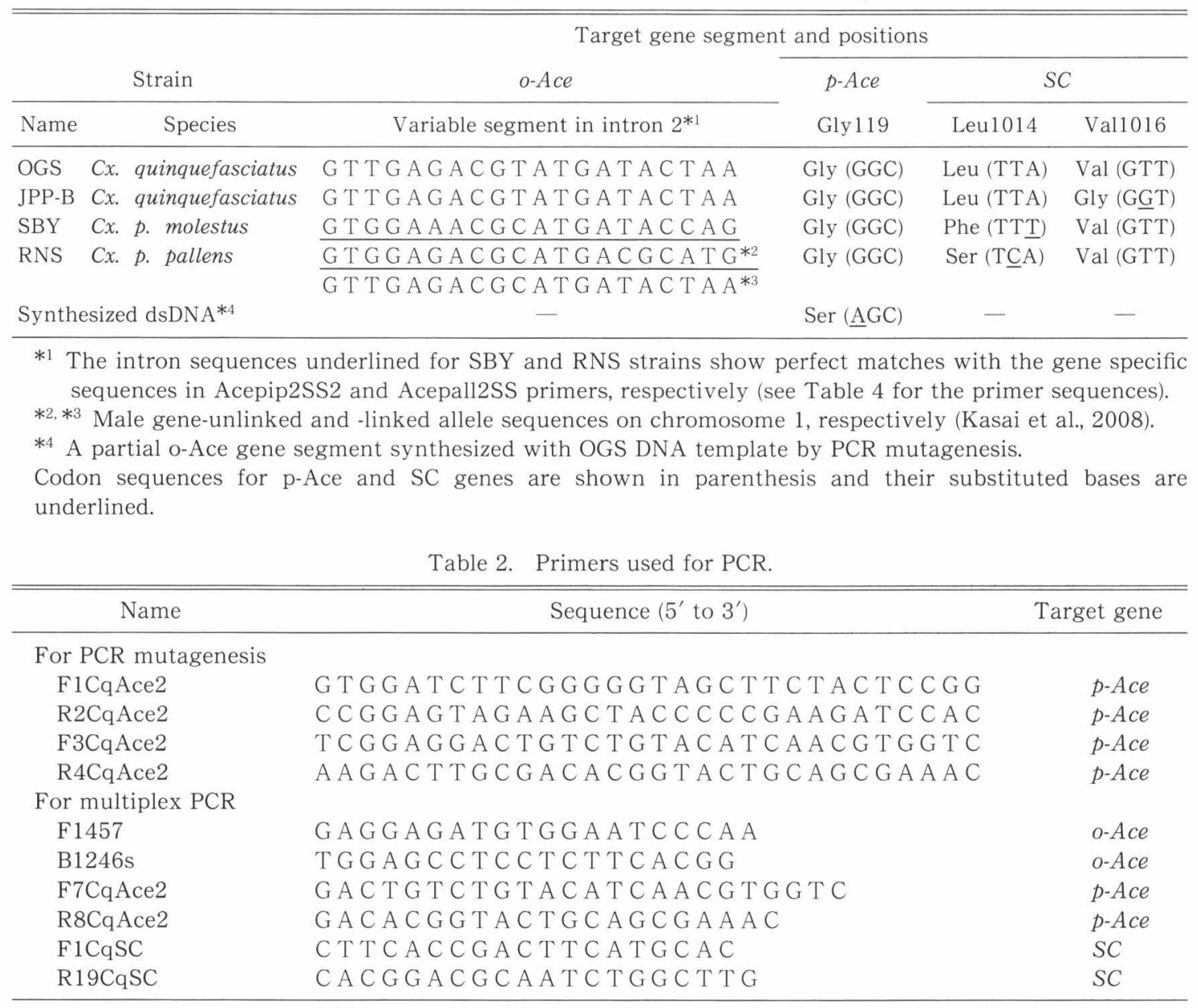

was synthesized based on the substitution in Cx. pipiens (GenBank\# AJ489456) by PCR mutagenesis. Two contiguous fragments were separately amplified with a primer set of $\mathrm{F} 3 \mathrm{CqAce} 2$ and $\mathrm{R} 2 \mathrm{CqAce} 2$ and another primer set of $\mathrm{F} 1 \mathrm{CqAce} 2$ and R4CqAce2 using the genomic DNA from OGS mosquitoes and then the second PCR was conducted using purified first PCR products and $\mathrm{F} 3 \mathrm{CqAce} 2$ and $\mathrm{R} 4 \mathrm{CqAce} 2$ primers. The sequences of the PCR primers are shown in Table 2.

\section{Molecular analysis}

Mosquito DNA was individually extracted with REDExtract-N-AmpTissue PCR Kit (Sigma-Aldrich) following the manufacturer's instructions with a half
Table 3. Constituents for multiplex PCR.

\begin{tabular}{lc}
\hline \hline \multicolumn{1}{c}{ Reagent } & Volume $(\mu l)$ \\
\hline dNTP $(2.5 \mathrm{mM}$ each $)$ & 2.00 \\
F1457 $(10 \mu \mathrm{M}$, primer for Ace 1$)$ & 0.80 \\
B1246S $(10 \mu \mathrm{M}$, primer for Ace 1$)$ & 0.80 \\
F7CqAce2 $(1 \mu \mathrm{M}$, primer for Ace 2$)$ & 2.00 \\
R8CqAce2 $(1 \mu \mathrm{M}$, primer for $A c e 2)$ & 2.00 \\
F1CqSC $(10 \mu \mathrm{M}$, primer for $S C)$ & 1.00 \\
R19CqSC $(10 \mu \mathrm{M}$, primer for $\mathrm{SC})$ & 1.00 \\
DNA solution & 0.50 \\
10X ExTaq buffer & 2.50 \\
ExTaq DNA polymerase $(0.5 \mathrm{U} / \mu l)$ & 0.25 \\
$\mathrm{H}_{2} \mathrm{O}$ & 12.15 \\
\hline Total & 25.00 \\
\hline
\end{tabular}

scale use of each reagent. The extracted DNA was finally collected in $112 \mu \mathrm{l}$ of neutralized solution and then stored at 
Table 4. SNaPshot primers and expected sequence variations.

\begin{tabular}{|c|c|c|c|c|c|c|c|}
\hline \multirow[b]{2}{*}{ Name } & \multirow{2}{*}{$\begin{array}{l}\text { Primer } \\
\text { Sequence }\end{array}$} & \multirow[b]{2}{*}{ Mobility* } & \multirow[b]{2}{*}{ Direction } & \multicolumn{2}{|c|}{ Target } & \multirow{2}{*}{$\begin{array}{l}\text { SNaPshot } \\
\text { signal }\end{array}$} & \multirow{2}{*}{$\begin{array}{l}\text { Estimated variations } \\
\text { (corresponding codons) }\end{array}$} \\
\hline & & & & Gene & Site & & \\
\hline Acepall2SS & $(\text { gact })_{10}$ G T G G A G A C G C A T G A C G C A T & 6 & forward & 0 -Ace & intron 2 & $\mathrm{~N}$ or none & any signal for $C x . p$. pallens \\
\hline Acepip2SS2 & 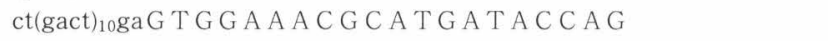 & 7 & forward & 0 -Ace & intron 2 & $\mathrm{~N}$ or none & any signal for $C x$. p. molestus \\
\hline R202CqAce1 & $(\text { gact })_{12} g$ C G C C A G T A T T T C T G C G T T G T A A A & 8 & reverse & $o$-Ace & intron 2 & $\mathrm{~N}$ & any signal for all subspecies \\
\hline R5CqAce2 & C A G T C C C G GA G T A GA A G C & 1 & reverse & p-Ace & Gly119 & $\mathrm{C}$ or $\mathrm{T}$ & Gly $(\underline{\underline{G G C}})$ or $\operatorname{Ser}(\underline{\underline{A G C}})$ \\
\hline R103CqSC & ct(gact) $)_{3} g a C$ A A A G C A A G G C T A A G A A A A G G T T A A G A & 3 & reverse & $S C$ & Val1016 & $\mathrm{A}$ or $\mathrm{C}$ & $\operatorname{Val}(\overline{\mathrm{G}} \underline{\underline{T} \mathrm{~T}})$ or $\mathrm{Gly}(\overline{\mathrm{G}} \underline{\underline{G}} \underline{\underline{T}})$ \\
\hline F108CqSC & $\operatorname{tgactgac}$ T G GCCAC C G TAGTGA TAGGAAAT $\underline{T}$ & 2 & forward & $S C$ & Leu1014 & $\mathrm{T}$ or $\mathrm{C}$ & Leu (TTR), Phe (TTY $)$, or Ser (TCN) \\
\hline F109CqSC & $(\text { (gact })_{6}$ G G C C A C C G T A G T G A T A G G A A A T T T & 4 & forward & $S C$ & Leu1014 & $\mathrm{R}, \mathrm{Y}$, or none & Leu $(\underline{T T} \underline{\underline{R}})$ or Phe $(\underline{\mathrm{TT}} \underline{\underline{\underline{Y}}})$ \\
\hline F110CqSC & 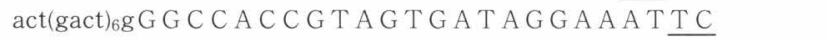 & 5 & forward & $S C$ & Leu1014 & $\mathrm{N}$ or none & $\operatorname{Ser}(\overline{\mathrm{TCN}})$ \\
\hline
\end{tabular}

* Ranks in electrophoretic mobility are in fast to slow order.

Lower case sequences denote nonspecific 5 '-tail repeats of the primers.

Bases with single underlines in 'Sequence' and 'Estimated variations' columns overlap within paired primer and template sets.

Bases with double underlines denote target variations. 
$-20^{\circ} \mathrm{C}$. Three DNA fragments of o-Ace, p-Ace and SC genes were amplified by multiplex PCR using the respective primer sets (Table 2) on a thermal cycler, MyCycler (Bio-Rad). The constituents of the PCR solution are shown in Table 3, where ExTaq (Takara) was used as DNA polymerase. The cycling conditions were preheating at $94^{\circ} \mathrm{C}$ for $2 \mathrm{~min}, 35$ cycles of $94^{\circ} \mathrm{C}$ for $30 \mathrm{sec}, 68^{\circ} \mathrm{C}$ for $30 \mathrm{sec}$ and $68^{\circ} \mathrm{C}$ for $60 \mathrm{sec}$, and finally elongation at $72^{\circ} \mathrm{C}$ for $5 \mathrm{~min}$. Unincorporated dNTPs and PCR primers were degraded with ExoSAP-IT (GE Healthcare) following the manufacturer's instructions.

The ExoSAP-IT-treated DNA solution was subjected to analysis of sequence polymorphism using SNaPshot Multiplex Kit (ABI) where gene specific primers (Table 4) were extended by single nucleotidyl addition with fluorescently labeled dideoxyribonucleotide triphosphates (ddNTPs). The constituents of the SNaPshot reaction solution are shown in Table 5 and the reaction was performed on the same thermal cycler following the manufacturer's cycling instructions. Unincorporated labeled ddNTPs were dephosphorylated with shrimp alkaline phosphatase (SAP, GE Healthcare) by adding $1 \mu \mathrm{l}$ SAP solution into $10 \mu l \mathrm{SNaPshot} \mathrm{reaction} \mathrm{solution.}$ The SAP-treated DNA solution was incubated at $37^{\circ} \mathrm{C}$ for one hour followed by an inactivation step at $75^{\circ} \mathrm{C}$ for $15 \mathrm{~min}$.

One half microlitter of the DNA solution was mixed with $0.5 \mu l$ of GeneScan-120 LIZ size standard (ABI) and $9 \mathrm{~m} l$ of Hi-Di formamide (ABI) and then the mixture was heated at $95^{\circ} \mathrm{C}$ for 2 min before electrophoresis. Capillary electrophoresis was performed in a $36 \mathrm{~cm}$ capillary with POP-7 polymer on an ABI PRISM 3130 Genetic Analyzer (ABI). The fluorescent signals were analyzed by GeneMapper v3.0 program (ABI).

\section{Laboratory colonies}

The localities of mosquito collection are given in Table 6. Larvae sampled at each of 19 collection sites in Honshu and
Table 5. Constituents of SNaPshot reaction solution.

\begin{tabular}{lc}
\hline \hline \multicolumn{1}{c}{ Reagent } & Volume $(\mu l)$ \\
\hline SNaPshot Ready Reaction Mix & 5.00 \\
R202CqAce1 $(10 \mu \mathrm{M})$ & 0.70 \\
Acepall2SS $(10 \mu \mathrm{M})$ & 0.02 \\
Acepip2SS2 $(10 \mu \mathrm{M})$ & 0.02 \\
R5CqAce2 $(10 \mu \mathrm{M})$ & 0.02 \\
F108CqSC $(10 \mu \mathrm{M})$ & 0.20 \\
F109CqSC $(10 \mu \mathrm{M})$ & 0.75 \\
F110CqSC $(10 \mu \mathrm{M})$ & 0.20 \\
R103CqSC $(10 \mu \mathrm{M})$ & 0.05 \\
Exo-SAP-treated PCR product & 1.00 \\
$\mathrm{H}_{2} \mathrm{O}$ & 2.04 \\
\hline Total & 10.00 \\
\hline
\end{tabular}

Kyusyu districts of Japan in 2004 and 2005 were reared in an insectarium and emerged females were tested for autogenicity by oviposition without bloodfeeding. From these females, autogenous and unautogenous colonies were started and several fourth instar larvae from each colony were subjected to molecular analysis within two generations after the colonization. Larvae sampled from several colonies were the survivors after selection with etofenprox (5.7 ppm) alone or etofenprox $(0.01 \mathrm{ppm})$ synergized with piperonyl butoxide $(0.5 \mathrm{ppm})$. The corresponding colonies are shown in Table 6.

\section{RESULTS}

\section{PCR design}

Multiplex PCR was designed to amplify DNA fragments of o-Ace, p-Ace and SC genes. The $o$-Ace PCR product included the sequence of intron 2 in its center and a slight variation in the product size ranging from $707-714 \mathrm{bp}$ is exhibited by the published 6 allele sequences from $C x$. $p$. pipiens, Cx. p. m., Cx. p. pallens and $C x . q$. (Aspen and Savage, 2003; Kasai et al., 2008). The PCR product for $p$-Ace was derived from a single exon (exon 5 ) and the product size expected was $174 \mathrm{bp}$ (Weill et al., 2003). The amplified SC gene fragment is split by intron 2 (Davis et al, 2007). This fragment exhibited a higher 
Table 6. Results of SNaPshot assay for biotyped recent laboratory mosquito colonies.

\begin{tabular}{|c|c|c|c|c|c|c|c|c|}
\hline \multirow{3}{*}{ Biotype } & \multicolumn{3}{|c|}{ Laboratory colony } & \multicolumn{5}{|c|}{ Judgement } \\
\hline & \multirow{2}{*}{ Code } & \multirow{2}{*}{ Origin } & \multirow{2}{*}{$\begin{array}{l}\text { No. } \\
\text { tested }\end{array}$} & \multirow{2}{*}{$\begin{array}{c}o-A c e \\
\text { intron } 2^{* 1}\end{array}$} & \multirow{2}{*}{$\begin{array}{c}p-A c e \\
\text { Gly } 119 \text { (GGC) }\end{array}$} & \multicolumn{2}{|c|}{$S C$} & \multirow{2}{*}{$\begin{array}{l}\text { No. } \\
\text { judged }\end{array}$} \\
\hline & & & & & & Leu1035 (TTA) & Val1037 (GTT) & \\
\hline \multirow[t]{13}{*}{ autogenous } & STM $1 * 2$ & Hatogaya City, Saitama Pref. & 4 & M & Gly (GGC) & Phe (TTT) & $\operatorname{Val}\left(\_\mathrm{TT}\right)$ & 4 \\
\hline & CHB & Chuou-ku, Chiba City, Chiba Pref. & 4 & M & Gly (GGC) & Phe (TTT) & $\operatorname{Val}\left(\_\mathrm{TT}\right)$ & 4 \\
\hline & TKY $1^{* 2}$ & Chiyoda-ku, Tokyo & 8 & M & Gly (GGC) & Phe (TTT) & Val (_TT) & 8 \\
\hline & TKY2 & Bunkyo-ku, Tokyo & 4 & M & Gly (GGC) & Leu (TTA) & Val (_TT) & 4 \\
\hline & TKY3 & Shinjuku-ku, Tokyo & 4 & M & Gly (GGC) & Leu (TTA) & Val (_TT) & 4 \\
\hline & TKY4 & Shinjuku-ku, Tokyo & 2 & M & Gly (GGC) & Leu (TTA) & Val (_TT) & 2 \\
\hline & TKY5 & Musashino, Tokyo & 3 & M & Gly (GGC) & Leu (TTA) & Val (_TT) & 3 \\
\hline & KNG & Kawasaki-ku, Kawasaki City, Kanagawa Pref. & 4 & M & Gly (GGC) & Leu (TTA) & Val (_TT) & 4 \\
\hline & $\mathrm{SZO}$ & Aoi-ku, Shizuoka City, Shizuoka Pref. & 4 & M & Gly (GGC) & Leu (TTA) & Val (_TT) & 4 \\
\hline & OSK & Chuou-ku, Osaka City, Osaka Pref. & 4 & $\mathrm{M}$ & Gly (GGC) & Leu (TTA) & Val ( TT) & 3 \\
\hline & & & & $\mathrm{M} / \mathrm{P}$ & Gly (GGC) & Leu (TTA) & Val (_TT) & 1 \\
\hline & $\mathrm{FKO}^{* 2}$ & Sawara-ku, Fukuoka City, Fukuoka Pref. & 4 & M & Gly (GGC) & Phe (TTT) & Val (_TT) & 4 \\
\hline & NGS1 & Nagasaki City, Nagasaki Pref. & 4 & M & Gly (GGC) & Leu $(\mathrm{TT} \overline{\mathrm{A}})$ & Val (_TT) & 4 \\
\hline \multirow[t]{8}{*}{ unautogenous } & STM2 & Kasukabe City, Saitama Pref. & 2 & $\mathrm{P}$ & Gly (GGC) & Leu (TTA) & Val (_TT) & 2 \\
\hline & $\mathrm{CHB} 2 * 2$ & Yokoshiba-cho, Sanbu-gun, Chiba Pref. & 4 & $\mathrm{P}$ & Gly (GGC) & Leu (TTA) & Val (_TT) & 4 \\
\hline & TKY $5 * 3$ & Shinagawa-ku, Tokyo & 5 & $\mathrm{P}$ & Gly (GGC) & Leu (TTA) & $\operatorname{Val}\left(\_\mathrm{TT}\right)$ & 2 \\
\hline & & & & & Gly (GGC) & $\begin{array}{c}\text { Leu/Ser } \\
\text { (TTA/TCA) }\end{array}$ & Val (_TT) & 3 \\
\hline & $\mathrm{AIC}^{* 2}$ & Higashi-ku, Nagoya City, Aichi Pref. & 4 & $\mathrm{P}$ & Gly (GGC) & Leu (TTA) & $\operatorname{Val}\left(\_\mathrm{TT}\right)$ & 4 \\
\hline & NGS1 & Nagasaki City, Nagasaki Pref. & 4 & $\mathrm{P}$ & Gly (GGC) & Leu (TTA) & $\operatorname{Val}\left(\_\mathrm{TT}\right)$ & 4 \\
\hline & NGS2 & Nagasaki City, Nagasaki Pref. & 4 & $\mathrm{P}$ & Gly (GGC) & Leu (TTA) & $\operatorname{Val}\left(\_\mathrm{TT}\right)$ & 4 \\
\hline & NGS3 & Nagasaki City, Nagasaki Pref. & 4 & $\mathrm{P}$ & Gly (GGC) & Leu (TTA) & Val (_TT) & 4 \\
\hline
\end{tabular}

$* 2$, *3 Larvae survived after insecticidal selection with etofenprox alone $(5.7 \mathrm{ppm})$ and etofenprox $(0.01$ ppm) synergized with piperonyl butoxide $(0.5$ ppm), respectively.

Substituted bases are underlined.

Blank bases with underbars are not determined from SNaPshot assay. 


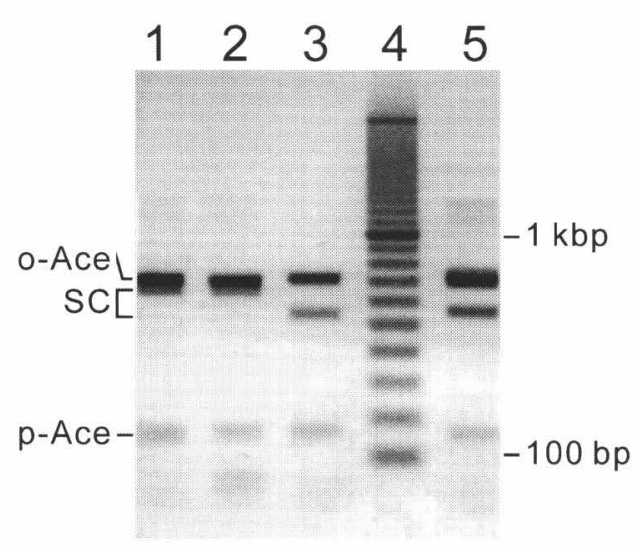

Fig. 1. Electrophoretic separation of multiplex PCR products. Lanes $1-3$ and 5, PCR products amplified with individual mosquitoes from JPP, OGS, SBY and RNS strains, respectively; lane 4, 100 bp DNA ladder.

size variation of between $553 \mathrm{bp}$ and 680 bp in 21 gene sequences from three $C x$. $p$. subspecies excluding $C x$. p. pipiens that was due to variability in the inserted intron size (Kasai, unpublished). The two target positions, Leu1014 and Val1016, in $\mathrm{SC}$ were split by this intron. The concentrations of the three primer sets were arranged to give similar molar products for the respective genes (Table 3 ) using four standard laboratory strains (Table 1). Examples of the gel electrophoretic separation for multiplex PCR products of the four strains are shown in Fig. 1.

\section{SNaPshot design}

SNaPshot reaction was designed to identify insecticide resistance-associated SNPs and mosquito subspecies by targeting o-Ace, p-Ace and SC genes (Fig. 2). In order to electrophoretically discriminate SNaPshot primers, 5 '-tails with nonspecific 'GACT' repeats were tailed to the gene specific sequences of the respective SNaPshot primers (Table 4).

Two primers, Acepall2SS and Acepip2 SS2, were designed for typing subspecies of the Cx. pipiens complex. Acepall2SS was designed to exclusively hybridize to $o$-Ace intron 2 from $C x$. p. pallens and the primer elongated with any single ddNTP at the 3 '-end in the SNaPshot reaction, while Acepip2SS2 was set to exclusively hybridize to $o$-Ace intron 2 from $C x . p . m$. and $C x$. p. pipiens followed by single nucleotidyl extensions to the primer. On the other hand, the SNaPshot DNA template derived from $C x . q$. did not hybridize to these two primers and resulted in no primer extension in principle. The usage of the present Acepall2SS and Acepip2SS 2 primers was modeled after our previous study (Kasai et al., 2008), where Cx. p. subspecies were classified by single sided AS-PCR with closely resembling allele specific primers, ACEpall2 (GTGGAGACGCATGACGCAT, equivalent to the gene specific $3^{\prime}$-side of Acepall2SS) and ACEpip 2 (GGTGGAAACGCATGATACCAG, equivalent to the gene specific $3^{\prime}$-side of Acepip2SS2 except for an additional gene specific nucleotide at the 5'-end of ACEpip 2). Both the present and our preceding assays targeted the common sequence variations (insertions/deletions and base substitutions) and the subspecies-specificities of the former ACEpall2 and ACEpip2 primers were previously already validated (Kasai et al., 2008). R202CqAce1 primer was designed from a segment of the invariable sequence in $o$-Ace intron 2 (Aspen and Savage, 2003; Kasai et al., 2008) as a positive control for 0 -Ace amplification.

R5CqAce2 primer was set to complementarily identify the SNP at the first base of the Gly 119 triplet sequence in the p-Ace gene. R103CqSC primer also identified the SNP at the middle base of Val1016 triplet sequence in the SC gene. The remaining three SNaPshot primers in Table 4 identified the SNPs in the Leu 1014 triplet sequence of the SC gene. F108CqSC discriminated Ser from Leu and Phe by reading the middle base of the Leu1014 position. F109CqSC identified either Leu or Phe by reading the third base of the Leu1014 position, but extension did not occur with this primer in the Ser substitution due to mismatching of the primer's 3 '-end base. F110CqSC 


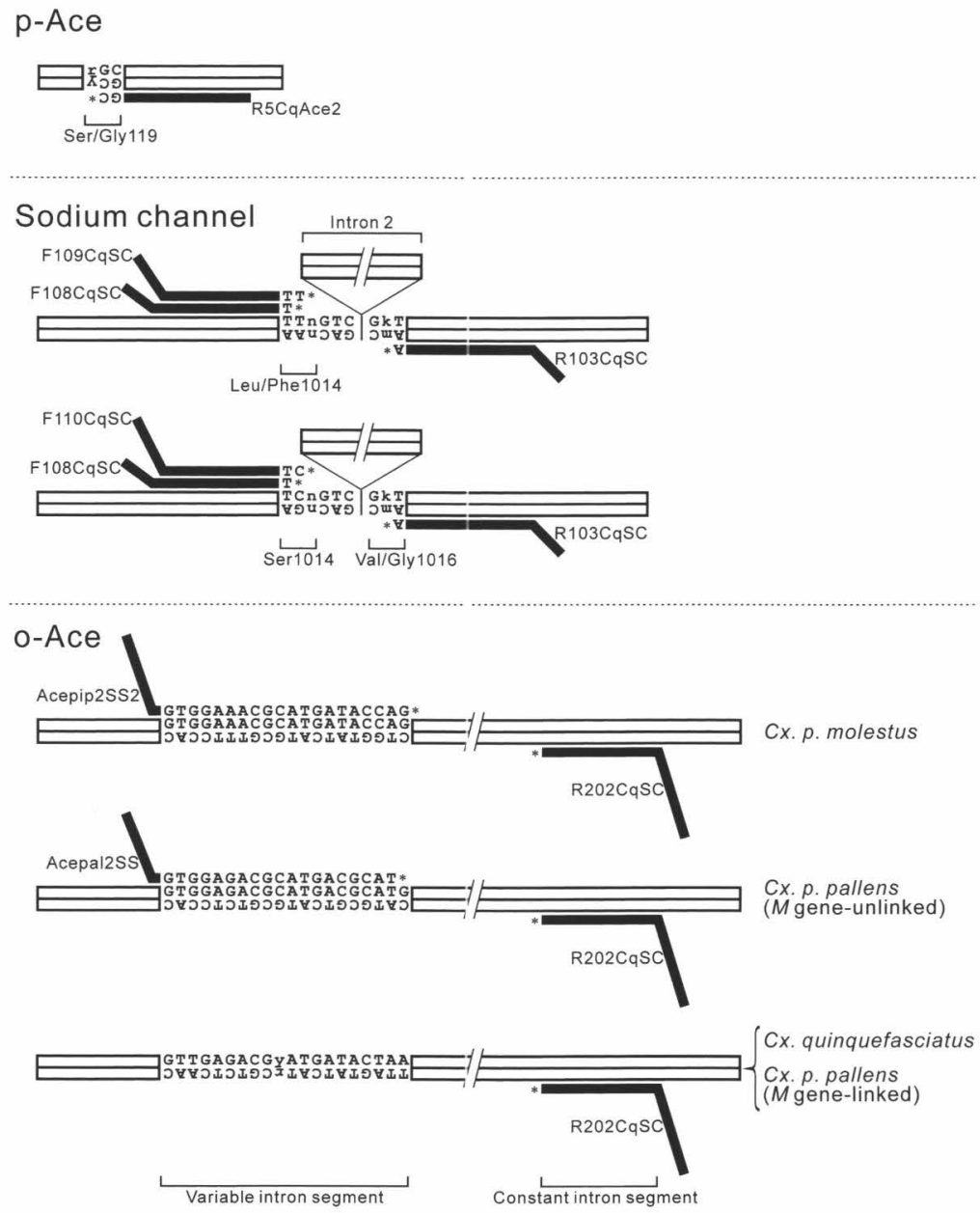

Fig. 2. Possible combinations of signaling primers with their respective target sequences in SNaPshot assay. Each black line and linked concrete nucleotide sequence as a unit indicates a SNaPshot primer. An asterisk indicates the fluorescence-labeled ddNTP that is added to a primer at the $3^{\prime}$-end in the SNaPshot reaction. Each white line split with concrete nucleotide sequence(s) indicates an amplified genomic DNA strand.

identified synonymous mutations in the Ser 1014 code, but no extension occurred with this primer in Leu or Phe also due to mismatching.

The concentrations of the SNaPshot primers were arranged to give rise to an allowable range of fluorescent signals (Table 3) with the multiplex PCR products from the standard laboratory strains (Table 1). The SNaPshot assay was performed using the synthesized $p$-Ace dsDNA as well as males from the standard strains. As a result, all electropherograms agreed with the predetermined DNA se- quences (Table 1). Examples of the electropherograms for the SBY strain of $C x$. $p$. $m$. and the RNS strain of $C x . p$. pallens are shown in Fig. 3.

As shown in the RNS strain (Table 1), the male of $C x$. p. pallens has a heterozygous allelic target sequence of $\mathrm{o}$-Ace intron 2 that is tightly linked to the maledetermining gene $(M)$ on chromosome 1 (Malcolm et al., 1998) and very similar to the sequence in $C x$. $q$. mosquitoes irrespective of sex (Kasai et al., 2008). There is a single base difference in the middle of the SNaPshot target segment between the $C x$. 


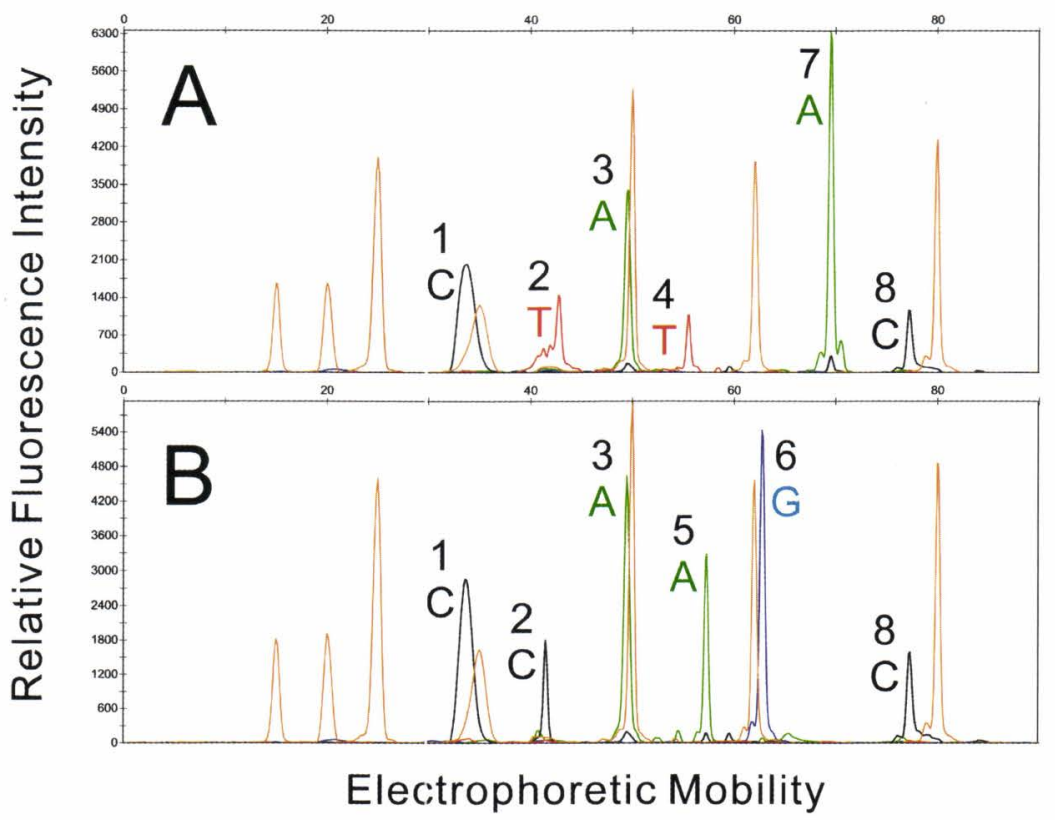

Fig. 3. Examples of SNaPshot electropherograms. A, SBY strain of Culex pipiens molestus; B, RNS strain of $C x$. p. pallens. Size standards of electrophoresis are shown in orange. Numbers and base codes beside peaks denote ranks in electrophoretic mobility of extended SNaPshot primers (see Table 4 for the corresponding primers) and base of the incorporated ddNTP, respectively.

$q$. sequence and the male-specific $C \%$. $p$. pallens sequence. However, the existence of the male specific sequence in $C x ; p$. pallens does not affect the present system or the previous AS-PCR-based system (Kasai et al., 2008). This rationale comes from the result that the $M$-linked chromosome gives rise to a null signal and the sole criterion is derived from the $M$ unlinked homologous chromosome in $C x$. p. pallens males (Kasai et al., 2008).

SNaPshot assay of recent laboratory colonies

Laboratory mosquitoes within two generations after field collection were tested for subspecies and resistance-associated mutations by SNaPshot analysis (Table 6). In 76 larvae sampled from the 19 colonies predetermined for autogenicity, all but one was judged either $C x . p . m$. or $C x . p$. pallens by SNaPshot assay. The results for the 75 individuals agreed not only with the autogenicity biotypes of the respective colonies but also with the subspe- cies determinded by the previous study that applied the AS-PCR method to molecular taxonomy (Kasai et al., 2008). The present study shares an $o$-Ace target segment and mosquito samples with the previous study. A single exceptional larva in the OSK colony was a hybrid of $C x . p . m$. and $C x$. p. pallens, possibly mistype because the same exceptional larva was typed as $C x$. p. $m$. by the AS-PCR assay (Kasai et al., 2008).

For the Gly 119 site in p-Ace, all samples were estimated to be homozygotes of the wild-type Gly (GGC), the a positive signal ensured the matching of reverse primer's 3 '-proximal to the 2 nd and $3 r$ d bases (GC) of the Gly code and the complementary ' $\mathrm{C}$ ' signal for the 1st base denoted ' $G$ ' in the positive-strand. For the Leu1014 site in SC, SNPs at the 2nd and 3rd bases were typed using three forward primers. The samples exhibited three doublets, 'TA', 'TT' and ' $(\mathrm{T} / \mathrm{C}) \mathrm{A}$ ' that represent the homozygotes of the wild-type Leu and mutant Phe, and the heterozygote of Leu and Ser, 
respectively. All the larvae tested from the insecticide-selected STM1, TKY1 and FKO colonies and the non-selected CHB colony of $C x . p . m$. were homozygous for Phe. Three of the 5 larvae tested in the insecticide-selected TKY 5 colony of $C x$. $p$. pallens were heterozygous for Ser. This is the first demonstration of typical and atypical $k d r$ mutants from $C x . p$. m. and $C x$. p. pallens populations in Japan, respectively. As for the Val1016 site in SC, the untargeted 1st base was ' $G$ ' in the wildtype Val (GTT) so all the samples were estimated to be homozygotes of $\mathrm{Val}$, where a positive signal ensured matching of the reverse primer's 3 '-end with the 3rd base (T) of the Val code and the complementary 'A' signal for the 2nd base denoted ' $\mathrm{T}$ ' in the positive-strand.

\section{DisCUSSION}

In common with the previous molecular taxonomic studies (Smith and Fonseca, 2004; Kasai et al., 2008), the present SNaPshot assay was unable to separate $C x . p . m$. from $C x$. $p$. pipiens because no interspecific variation between the two subspecies was available in mutual targetting the sequence of intron 2 in the o-Ace gene. However, this methodological limitation does not impair the practical significance of these studies in the census of populations in Japan and temperate and tropical regions of Asia for which no $C x$. $p$. pipiens have been previously identified (Sasa, 1971; Tanaka et al., 1979; Tanaka, 2005).

In this study, the Phe and Ser mutations at the Leu1014 site were identified in $C x$. p. $m$. and $C x$. p. pallens, respectively. The Ser mutation was originally found in a pyrethroid resistant strain of $C x . p$. mosquitoes (Martinez-Torres et al., 1999). The significance of this mutation for pyrethroid resistance is supported from other studies by Kasai (unpublished) as follows: the RNS strain originally segregating Ser mutant alleles at 19\% were subjected to larval selection with etofenprox for 7 generations and the fre- quency finally reached 99\%, suggesting that the Ser1014 mutation in SC contributes to pyrethroid resistance by reduced nerve sensitivity. In accordance with the increase in the Ser mutant allele frequency, the selected RNS strain has acquired an extraordinarily high resistance at a $10^{3}$ in resistance ratio for etofenprox. However, increased microsome monooxygenase activity was also involved in the resistance mechanisms as well as an atypical Ser-type $k d r$ mutation.

Analogous pyrethroid resistance mechanisms (i.e. involving both elevated metabolism and reduced nerve sensitivity) are present in SBY and JPP-B strains, where Phe-type typical $k d r$ and Val1016 Gly $k d r$-like mutations in the SC genes are fixed, respectively, and $10^{3}$-fold etofenprox resistance were exhibited (Kasai, unpublished). The elevated metabolism in microsome monooxygenases is likely ascribed to overexpression of cytochrome P450 genes in these three strains (Komagata, unpublished). SNP-typing methods including SNaPshot assay generally have technical limitations in adaptation to the mutations that affect gene expression levels.

Highly developed resistance to several insecticide groups including pyrethroids has been demonstrated in local underground colonies of $C x . p . m$. in Japan, probably due to the activity of pest control industries (Kasai et al., 2007). To prevent WNF outbreaks, systematically enforced chemical control of the major mosquito vector, $C x$. $p$. group, is needed under the initiative of local governments. Under consistent selective pressure, insecticide resistant genes prevailing in $C x$. p. $m$. populations may potentially result in introgressive hybridization among $C x$. $p$. subspecies and may cause control problems in overground subspecies. If the subspecies specificities of the Phe and Ser mutations at Leu1014 in SC are more rigidly confirmed in the $C x . p . m$. population by further investigations, we can evaluate the possible occurrence of introgression by 
targeting these mutations and their marginal haplotypes. An analogous SNaPshot assay will serve as an important tool for such a research project.

Molecular genotyping techniques have been developed based on thermal-cycling or isothermic reactions for amplifying allelic targets or signals to identify insecticide resistance in medically and agriculturally important pests: e.g. organophosphate and carbamate insensitive AChE genes of the cotton aphid by AS-PCR assay (Toda et al., 2008); $k d r$ mutations of the mosquito Anopheles gambiae by hot ligation oligonucleotide assay (HOLA) (Lynd et al., 2005); $k d r$-like mutations of the headlouse by invader assay (Kim et al., 2004) and TaqMan assay (Tomita et al., 2005). These assays detected of single or multiple specified bases at a target nucleotide position in a single reaction well. SNaPshot assay has an advantage over the above assays because it enables a single reaction well to detect any of four possible bases at each multiple target loci.

Recently after the onset of the present study, further significant genetic variations in molecular taxonomy and resistance-genotyping have been reported for $C x$. $p$. mosquitoes. Interspecific variations in CQ11 microsatellite locus discriminated $C x . p$. $m$. and $C x$. p. pipiens (Bahnck and Fonseca, 2006). A new amino acid substitution mutation, Phe290Val, in p-Ace that confers insecticide resistance was found in $C x$. p. mosquitoes from Cyprus (Alout et al., 2007). Our SNaPshotbased genotyping system will allow for flexible incorporation of these targets by adding new primer subsets for multiplex PCR and minisequencing.

\section{ACKNOWLEDGEMENTS}

This work was partly supported by Grant-in-Aids for Scientific Research from the Ministry of Health, Labor and Welfare (H16-Kenko-ippan-052 to IT and TT) and (H18-Shinkou-ippan-018 to MK), and for Young Scientists (A) from the Ministry of Education, Culture, Sports, Science and Technology to SK (17790281). O. Komagata is a Research Resident of Japan Health Science Foundation.

\section{REFERENCES}

Alout, H., Berthomieu, A., Hadjivassilis, A. and Weill, M. 2007. A new amino-acid substitution in acetylcholinesterase 1 confers insecticide resistance to Culex pipiens mosquitoes from Cyprus. Insect Biochem. Mol. Biol., 37: 41-47.

Aspen, S. and Savage, H. 2003. Polymerase chain reaction assay identifies North American members of the Culex pipiens complex based on nucleotide sequene differences in the acetylcholinesterase gene Ace.2. J. Am. Mosq. Control Assoc., 19: 323328.

Bahnck, C. M. and Fonseca, D. M. 2006. Rapid assay to identify the two genetic forms of Culex (Culex) pipiens L. (Diptera: Culicidae) and hybrid populations. Am. J. Trop. Med. Hyg., 75: 251-255.

Bourguet, D., Fonseca, D., Vourch, G., Dubois, M., Chandre, F., Severini, C. and Raymond, M. 1998. The acetylcholinesterase gene Ace: a diagnostic marker for the pipiens and quinquefasciatus forms of the Culex pipiens complex. J. Am. Mosq. Control Assoc., 14: 390-396.

Brengues, C., Hawkes, N. J., Chandre, F., McCarroll, L., Duchon, S., Guillet, P., Manguin, S., Morgan, J. C. and Hemingway, J. 2003. Pyrethroid and DDT cross-resistance in Aedes aegypti is correlated with novel mutations in the voltage-gated sodium channel gene. Med. Vet. Entomol., 17: 87-94.

Casimiro, S., Coleman, M., Mohloai, P., Hemingway, J. and Sharp, B. 2006. Insecticide resistance in Anopheles funestus (Diptera: Culicidae) from Mozambique. J. Med. Entomol., 43: 267-275.

Cui, F., Raymond, M. and Qiao, C. L. 2006. Insecticide resistance in vector mosquitoes in China. Pest Manag. Sci., 62: 1013-1022.

Davies, T. G. E., Field, L. M., Usherwood, P. N. R. and Williamson, M. S. 2007. A comparative study of voltage-gated sodium channels in the Insecta: implications for pyrethroid resistance in Anopheline and other Neopteran species. Insect Mol. Biol., 16: 361-375.

Etang, J., Fondjo, E., Chandre, F., Morlais, I., Brengues, C., Nwane, P., Chouaibou, M., Ndjemai, H. and Simard, F. 2006. First report of knockdown 
mutations in the malaria vector Anopheles gambiae from Cameroon. Am. J. Trop. Med. Hyg., 74: 795797.

Ferre, J. and Van Rie, J. 2002. Biochemistry and genetics of insect resistance to Bacillus thuringiensis. Ann. Rev. Entomol., 47: 501-533.

Hamadan, H., Sofian-Aziron, M., Nazni, W. A. and Lee, H. L. 2005. Insecticide resistance development in Culex quinquefasciatus (Say), Aedes aegypti (L.) and Aedes albopictus (Skuse) larvae against malathion, permethrin and temephos. Trop. Biomed., 22: 45-52.

Harbach, R., Harrison, B. and Gad, A. 1984. Culex (Culex) molestus Forskal (Diptera: Culicidae): neotype designation, description, variation, and taxonomic status. Proc. Entomol. Soc. Wash., 86: 521542.

Ishii, T. 1978. Some problems on Culex pipiens molestus in Japan. Akaieka Newsl., 3: 1-14.

Kasai, S., Komagata, O., Tomita, T., Sawabe, K., Tsuda, Y., Kurahashi, H., Ishikawa, T., Motoki, M., Takahashi, T., Tanikawa, T., Yoshida, M., Shinjo, G., Hashimoto, T., Higa, Y. and Kobayashi, M. 2008. PCR-based identification of Culex pipiens complex collected in Japan. Jpn. J. Infect. Dis., 61: 184-191.

Kasai, S., Shono, T., Komagata, O., Tsuda, Y., Kobayashi, M., Motoki, M., Kashima, I., Tanikawa, T., Yoshida, M., Tanaka, I., Shinjo, G., Hashimoto, T., Ishikawa, T., Higa, Y. and Tomita, T. 2007. Insecticide resistance in potential vector mosquitoes for West Nile virus in Japan. J. Med. Entomol., 44: 822-829.

Kasai, S., Weerasinghe, I. S. and Shono, T. 1998. P450 monooxygenases are an important mechanism of permethrin resistance in Culex quinquefasciatus Say larvae. Arch. Insect Biochem. Physiol., 37: 4756.

Kawakami, Y. 1989. Insecticide-resistance of Culex pipiens molestus Forskal collected in Shinjuku-ku, Tokyo. Jpn. J. Sanit. Zool., 40: 217-220.

Kim, H. J., Symington, S. B., Hyeock Lee, S. and Clark, J. M. 2004. Serial invasive signal amplification reaction for genotyping permethrin-resistant ( $k d r$ like) human head lice, Pediculus capitis. Pestic. Biochem. Physiol., 80: 173-182.

Kono, Y. and Tomita, T. 1992. Characteristics of highly active carboxylesterases in insecticideresistant Culex pipiens quinquefasciatus. Med. Entomol. Zool., 43: 297-305.

Lynd, A., Ranson, H., McCall, P. J., Randle, N. P., Black, W. C., Walker, E. D. and Donnelly, M. J. 2005. A simplified high-throughput method for pyrethroid knock-down resistance $(k d r)$ detection in Anopheles gambiae. Malar. J., 4: 16.

Malcolm, C. A., Bourguet, D., Ascolillo, A., Rooker, S. J., Garvey, C. F., Hall, L. M., Pasteur, N. and Raymond, M. 1998. A sex-linked Ace gene, not linked to insensitive acetylcholinesterase-mediated insecticide resistance in Culex pipiens. Insect. Mol. Biol., 7: 107-120.

Martinez-Torres, D., Foster, S. P., Field, L. M., Devonshire, A. L. and Williamson, M. S. 1999. A sodium channel point mutation is associated with resistance to DDT and pyrethroid insecticides in the peach-potato aphid, Myzus persicae (Sulzer) (Hemiptera: Aphididae). Insect. Mol. Biol., 8: 339346.

Miyazaki, M., Ohyama, K., Dunlap, D. Y. and Matsumura, F. 1996. Cloning and sequencing of the para-type sodium channel gene from susceptible and $k d r$-resistant German cockroaches (Blattella germanica) and house fly (Musca domestica). Mol. Gen. Genet., 252: 61-68.

Nazni, W. A., Lee, H. L. and Azahari, A. H. 2005. Adult and larval insecticide susceptibility status of Culex quinquefasciatus (Say) mosquitoes in Kuala Lumpur Malaysia. Trop. Biomed., 22: 63-68.

Sasa, M. 1971. Species problems in Culex pipiens and Cx. tritaeniorhynchus. In: Progress in Medical Zoology I (ed. Sasa, M.), pp. 49-65, Gakujutsusho Shuppankai, Tokyo.

Sharma, S. N., Saxena, V. K. and Lal, S. 2004. Study on susceptibility status in aquatic and adult stages of Aedes aegypti and Ae. albopictus against insecticides at international airports of south India. $J$. Commun. Dis., 36: 177-181.

Smith, J. and Fonseca, D. 2004. Rapid assays for identification of members of the Culex (Culex) pipiens complex, their hybrids, and other sibling species. Am. J. Trop. Med. Hyg., 70: 339-345.

Tanaka, K. 2005. Culicidae. In: Aquatic Insects of Japan: Manual with Keys and Illustrations (ed. Kawai, T. and Tanida, K.), pp. 757-1006, Tokai University Press, Kanagawa.

Tanaka, K., Mizusawa, K. and Saugstad, E. 1979. A revision of the adult and larval mosquitoes of Japan (including the Ryukyu archipelago and the Ogasawara islands) and Korea (Diptera: Culicidae). Contrib. Am. Entomol. Inst., 16: 1-987.

Tia, E., Akogbeto, M., Koffi, A., Toure, M., Adja, A. M., Moussa, K., Yao, T., Carnevale, P. and Chandre, E. 
2006. Pyrethroid and DDT resistance of Anopheles gambiae s.s. (Diptera: Culicidae) in five agricultural ecosystems from Cote-d'Ivoire. Bull. Soc. Pathol. Exot., 99: 278-282.

Toda, S., Komazaki, S., Izawa, H., Nakada, K., Kanazaki, S., Souda, E. and Shigehara, T. 2008. Development of molecular diagnostics of the two point mutations in acetylcholinesterase gene associated insecticide resistance in the cotton aphid, Aphis gossypii Glover (Homoptera: Aphididae), and a survey of genotypic frequency in field population. Appl. Entomol. Zool., 43: 127133.

Tomita, T., Yaguchi, N., Mihara, M., Agui, N. and Kasai, S. 2005. Sodium channel point mutations associated with pyrethroid-resistance in the head louse. In: New Discoveries in Agrochemicals (ed. Clark, J. M. and Ohkawa, H.), pp. 234-243, Oxford University Press, Washington, D. C.

Vatandoost, H., Hashavekhi, M., Abaie, M. R., Aflatoonian, M. R., Hanafi-Bojd, A. A. and Sharifi, I. 2005. Monitoring of insecticides resistance in main malaria vectors in a malarious area of Kahnooj district, Kerman province, southeastern Iran. $J$. Vector Borne Dis., 42: 100-108.

Vinogradova, E. 2000. Culex pipiens pipiens Mosquitoes: Taxonomy, Distribution, Ecology, Physiology, Genetics, Applied Importance and Control. 250 pp., Pensoft Publishers, Moscow.

Weill, M., Lutfalla, G., Mogensen, K., Chandre, F., Berthomieu, A., Berticat, C., Pasteur, N., Philips, A., Fort, P. and Raymond, M. 2003. Comparative genomics: Insecticide resistance in mosquito vectors. Nature, 423: 136-137.

Williamson, M. S., Martinez-Torres, D., Hick, C. A. and Devonshire, A. L. 1996. Identification of mutations in the housefly para-type sodium channel gene associated with knockdown resistance $(k d r)$ to pyrethroid insecticides. Mol. Gen. Genet., 252: 5160.

Xu, Q., Liu, H., Zhang, L. and Liu, N. 2005. Resistance in the mosquito, Culex quinquefasciatus, and possible mechanisms for resistance. Pest. Manag. Sci., 61: 1096-1102. 